\title{
Le prix Nobel de physique 2020, ou la consécration des trous noirs
}

Nathalie Deruelle ${ }^{(1)}$ (deruelle@apc.univ-paris7.fr) et Jean-Pierre Lasota(2)

(1) Laboratoire Astroparticule et Cosmologie, Université Paris Diderot, Bâtiment Condorcet, 10 rue A. Domon et Léonie Duquet, 75205 Paris Cedex 13

(2) Institut d'Astrophysique de Paris, 98 bis Boulevard Arago, 75014 Paris

Professeur au Centre d'Astronomie Nicolas Copernic à Varsovie

Trois lauréats partagent le prix

Nobel de physique 2020

pour leurs découvertes

sur l'un des phénomènes

les plus exotiques de l'Univers,

les trous noirs :

le théoricien britannique Roger

Penrose, l'astronome allemand

Gerhard Genzel et l'astronome

américaine Andrea Ghez.
Albert Einstein reçut en 1922 le prix Nobel de 1921 «pour les services qu'il a rendus à la physique théorique, et particulièrement pour sa découverte de la loi de l'effet photoélectrique ». Dans son discours de présentation, Arrhenius mentionne aussi la théorie du mouvement brownien. Mais il expédie la théorie de la relativité en un paragraphe : «[...] Elle relève essentiellement de l'épistémologie et a en conséquence été l'objet de débats animés dans les cercles philosophiques. Ce n'est pas un secret que le célèbre philosophe de Paris Henri Bergson a mis en doute la théorie, quand d'autres philosophes l'ont acceptée sans réserve. La théorie en question a également des implications astrophysiques qui sont actuellement rigoureusement examinées. »

Le message est clair : le comité Nobel n'attribue pas ses prix aux bâtisseurs de théories, mais aux prédictions ou explications de faits expérimentaux ou observationnels. Ainsi, pas plus que la petite dizaine d'autres décernés au fil des années "autour " de la relativité générale - y compris celui de 2017 (attribué pour "des contributions décisives au détecteur LIGO et l'observation d'ondes gravitationnelles ") - pas plus donc que tous les autres, le prix Nobel de physique 2020, décerné à Roger Penrose, Reinhard Genzel et Andrea Ghez, ne consacre la théorie de la gravitation d'Einstein, encore moins sa vision révolutionnaire de l'espace et du temps.

Mais il s'en rapproche - en deux étapes.
Pour moitié, le prix Nobel de physique 2020 récompense Reinhard Genzel et Andrea Ghez "pour la découverte d'un objet compact supermassif au centre de notre galaxie ": le comité est resté prudent, le mot " trou noir " n'est pas prononcé dans la citation les concernant. Pourtant, que pourrait-ce être d'autre?

En 1963, Maarten Schmidt découvrait les formidables émetteurs d'énergie électromagnétique que sont les "quasars ", objets ponctuels lointains dont les luminosités sont comparables à celles de galaxies entières. En 1964, Edward Salpeter et, indépendamment, Yakov Zeldovich proposaient que leur source d'énergie était l'accrétion de matière sur des " objets effondrés ", baptisés peu après " trous noirs ". Et en 1969, Donald Lynden-Bell prédisait que des trous noirs de plusieurs millions ou dizaines de millions de masses solaires pouvaient être présents au centre des galaxies, reliques quasi-éteintes de quasars.

Depuis, une bonne dizaine de telles reliques ont été repérées, dont une, il y a une quarantaine d'années, appelée "Sagittarius $A \star ”\left(\operatorname{Sgr} A^{\star}\right)$, faible source radio au cœur de notre galaxie, la Voie lactée.

Pour pouvoir identifier cet objet particulier à un trou noir supermassif il fallait montrer... qu'il était compact, noir (l'émission radio provenant de sa zone proche) et très massif : tâche ardue.

SgrA * étant à 25000 années-lumière de la Terre, il a d'abord été possible, malgré la distance et l'opacité du centre galactique 


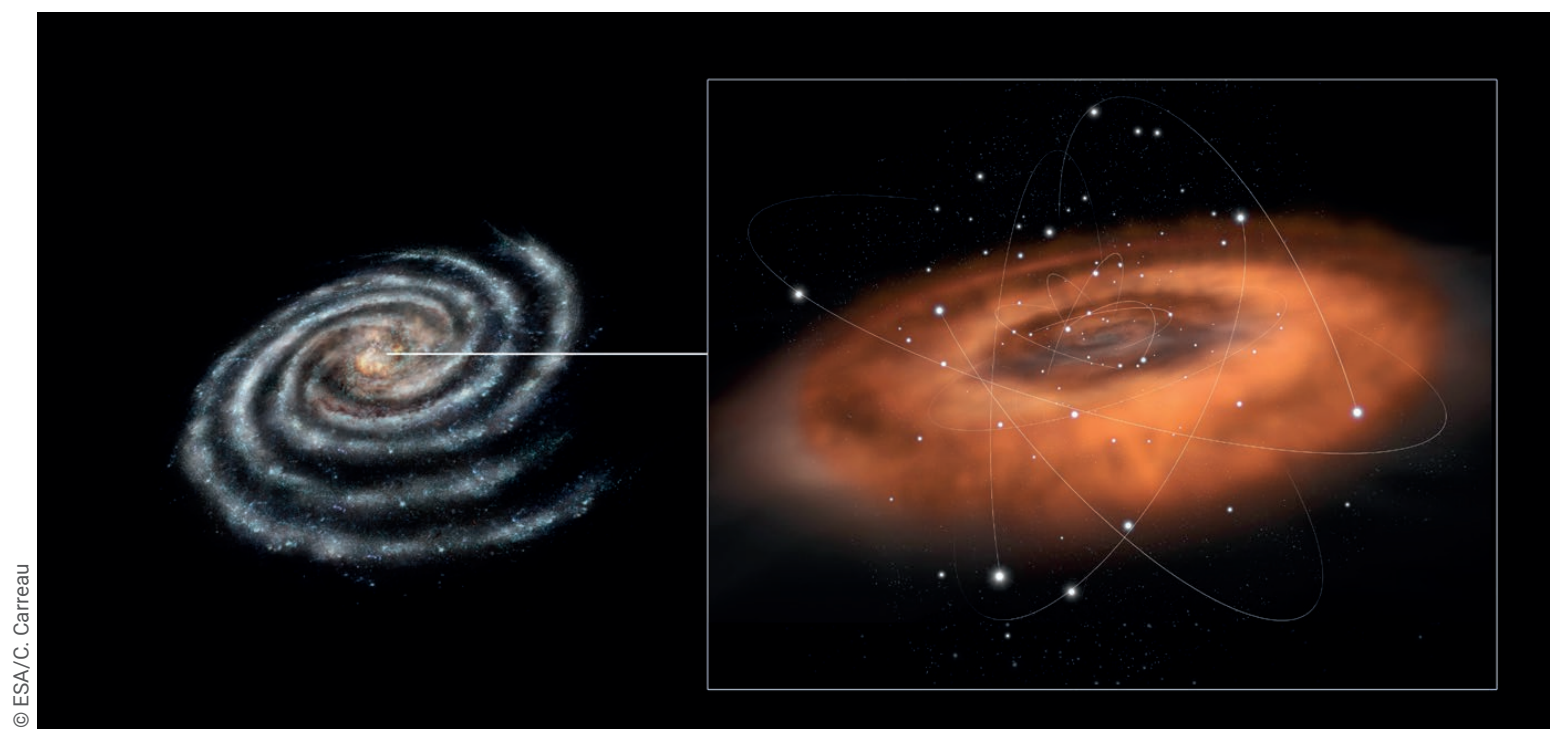

Montage de photos illustrant la région centrale de la Voie lactée. La photo de droite, prise par le satellite Herschel, montre un tore de gaz moléculaire et de poussières entourant le centre de notre galaxie, qui abrite le trou noir supermassif SgrA*.

qui limite les observations au domaine infrarouge, de repérer des étoiles ordinaires dans son voisinage. Il fallut ensuite suivre leurs mouvements autour de lui, vérifier que les orbites étaient en première approximation des ellipses pour en déduire par les lois de Kepler la masse de l'objet central. Et, en plus, il fallait vérifier que toutes les ellipses avaient le même foyer, pour exclure la possibilité que l'objet central ne fut quelque exotique amas de millions de corps sombres distincts.

Pour arriver à des conclusions solides, il fallut cependant attendre la révolution dite de "l'optique adaptative ", qui permet de corriger en temps réel les déformations des images dues à l'atmosphère terrestre. Il fallut aussi les consolider et les prolonger par un tour de force : celui de la collaboration européenne GRAVITY (dont le groupe de Genzel est leader, et à laquelle participent activement de nombreuses équipes françaises) qui réussit à coupler les quatre "très grands télescopes " (VLT) de l'ESO au Chili, de 8,5 mètres chacun, pour les transformer en un interféromètre dont la résolution est celle d'un télescope « effectif " de 130 mètres.

Grâce à leur persévérance de près de 30 ans, et grâce à ces progrès technologiques, l'équipe de Genzel (de l'Institut Max Planck de Garching près de Munich, MPE) et celle de Ghez (Université de Californie à Los Angeles, UCLA), utilisant pour la première les télescopes de l'ESO au Chili et, pour la seconde, les télescopes

\section{Biographies}

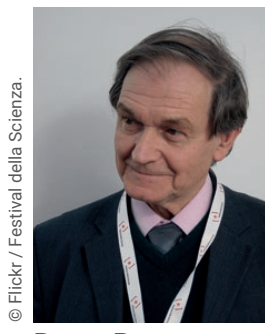

Roger Penrose en 2011.

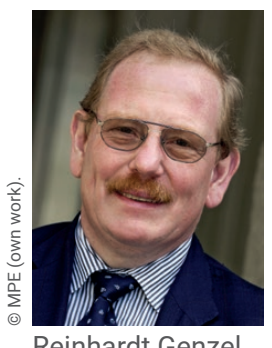

Reinhard Genzel est un astronome allemand, né en 1952. Depuis 2010, il est directeur à l'Institut Max-Planck de physique extraterrestre, à Garching près de Munich. Avec son équipe il se consacre principalement aux observations du centre de la Voie lactée à l'aide des télescopes de l'ESO au Chili. Ils ont été les premiers à mesurer précisément le mouvement des étoiles à sa proximité et en ont déduit la présence d'un trou noir supermassif de 4 millions de masses solaires. Ils ont aussi mis en évidence la présence de sursauts lumineux se produisant dans le voisinage immédiat du trou noir dans les longueurs d'onde infrarouge, ce qui donne la mesure la plus précise de la taille de l'objet compact au centre de la Voie lactée.

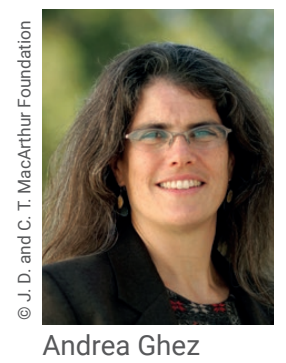

Andrea Mia Ghez est une astronome américaine, née en 1965. Elle est professeure au département de physique et d'astronomie de l'Université de Californie à Los Angeles. Avec son équipe, elle se consacre principalement aux observations du centre de la Galaxie à l'aide des télescopes Keck à Hawaï. Ghez et son équipe ont amélioré de façon significative la précision des observations du mouvement des étoiles du centre galactique obtenues auparavant par Genzel et son équipe. 
$>>$

Keck à Hawaï, ont relevé le défi. Leurs observations [1,2], en parfait accord, d'une dizaine d'étoiles gravitant autour de SgrA *, en particulier l'étoile S2 dont la période n'est que de seize ans et l'excentricité grande, sont sans appel, ainsi que le conclut (avec la prudence qui le caractérise) le comité Nobel dans l'article qui détaille les travaux des lauréats : "Ces observations fournissent une base solide à l'hypothèse que l'objet compact au centre de notre galaxie est un trou noir supermassif, tel que la théorie de la relativité générale les prédit. »

Mieux encore : la correction combinée de l'effet Doppler transverse et du décalage gravitationnel vers le rouge ainsi que l'avance du périastre (point de l'orbite le plus proche du foyer) ont été mesurées (cette dernière tout récemment), en accord avec les prédictions de la relativité générale (fig.1).

Le prix Nobel de physique 2020 récompense pour son autre moitié Roger Penrose "pour la découverte que la formation de trous noirs est une prédiction robuste de la théorie de la relativité générale " : pour la première fois, la théorie d'Einstein de la gravitation est reconnue, et la plus spécifique de ses prédictions, les trous noirs, explicitement mentionnée dans une citation de prix Nobel.

L'ironie de cette citation est qu'Einstein, on le sait, avait récusé ces " astres de Schwarzschild ", où le temps semblait s'arrêter et l'espace se déchirer sur leur surface. Ce n'est que dix ans après sa mort qu'ils furent dédiabolisés : on comprit définitivement que leur surface immatérielle n'avait rien de singulier, que la lumière y était seulement piégée par le champ de gravitation, formant pour nous comme un « horizon " masquant leur intérieur.

Il n'en restait pas moins qu'au centre d'un trou noir de Schwarzschild se niche une "vraie » singularité, où la gravitation est si forte que même l'espace et le temps ne peuvent plus y être définis. Même si cette singularité "de courbure " reste invisible au monde extérieur, on comprend qu'on ait voulu savoir si elle n'était pas qu'un artefact de la grande symétrie (sphérique en l'occurrence) du trou noir de Schwarzschild. (La même question se pose pour les trous noirs en rotation, dits de Kerr, à symétrie axiale.)

Et c'est ici que Penrose intervient.

Roger Penrose est mathématicien. Son article de 1965 [3], pour lequel il a obtenu le

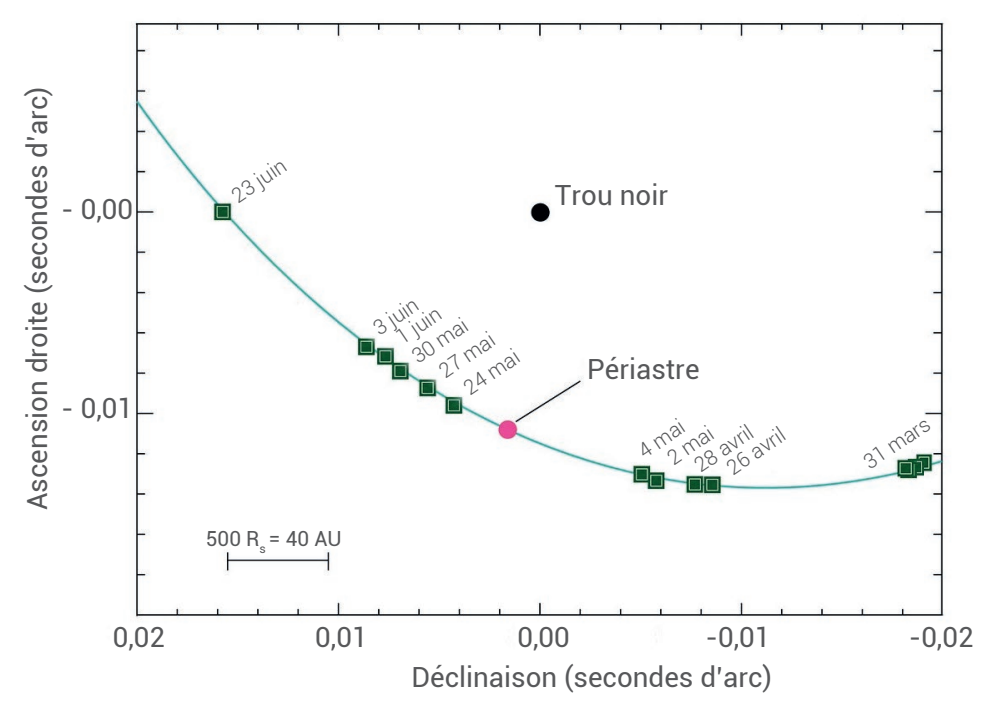

1. Orbite de l'étoile $S 2$ autour de SgrA*, en coordonnées équatoriales ascension droite et déclinaison (équivalentes sur la sphère céleste à la longitude et la latitude terrestres). SgrA* est un objet sombre, coïncidant avec une faible source radio, et très compact (sinon la trajectoire ne serait pas une ellipse, corrections relativistes incluses, projetée sur la sphère céleste). Sa masse est de 4,152 millions de masses solaires. C'est donc un trou noir supermassif. Le rayon $\mathrm{R}_{\mathrm{S}}$ de son horizon (c'est-à-dire la surface qui l'entoure d'où aucun objet ou rayon de lumière ne peut échapper à son champ gravitationnel), proportionnel au double de sa masse, est de 12 millions de km (soit seulement 17 fois celui du Soleil).

\section{Les apports de la topologie différentielle au théorème de Penrose}

Supposons que (voir la figure 2) :

(1) l'hyper-surface $C^{3}$ où sont données les conditions initiales déterminant partout l'évolution de la géométrie est « non compacte » (l'espace euclidien en est un exemple) ;

(2) les rayons de lumière issus de la surface piégée $T^{2}$ peuvent être prolongés ad infinitum. La gravité étant attractive et régie par les équations d'Einstein, la frontière $\mathrm{B}^{3}$ de la portion $\mathrm{F}^{4}$ de $\mathrm{M}^{4}$, ensemble des points dans le futur de $\mathrm{T}^{2}$, est alors fermée, compacte et sans bord. L'image de $\mathrm{B}^{3}$ dans $\mathrm{C}^{3}$ (par homéomorphisme) doit l'être aussi. Mais cela n'est pas plus possible que de plonger une 2-sphère dans un 2-plan (car on ne peut pas l'« aplatir »).

Par conséquent une des hypothèses du théorème est fausse. La plus « fragile » est (2). Donc : un rayon de lumière, au moins, issu de $\mathrm{T}^{2}$, doit cesser d'exister au-delà d'un certain point de sa trajectoire ; $\mathrm{M}^{4}$ présente une singularité.

On voit ainsi la splendeur et les misères de ce théorème.

Ses misères : il ne dit rien sur l'existence ou non d'un horizon qui cacherait ces points où la lumière s'arrête (donc, il ne prédit pas l'existence de trous « noirs »); il ne dit rien non plus sur la nature de la singularité (qui n'est pas nécessairement une singularité de courbure où tout diverge).

Sa splendeur : il est très général et suppose très peu de choses sur la matière ( $s^{\prime} i l$ y en a) et, surtout, il introduit ces fructueuses notions de topologie en relativité générale.

prix, intitulé « Effondrement gravitationnel et singularités d'espace-temps ", énonce donc un théorème.

Sa substantifique moelle est : si Einstein a raison ; si la densité de matière en effondrement (aussi asymétrique que l'on veut) est positive (ou, dit différemment, si la gravité est partout attractive) ; si l'évolution est entièrement déterminée à partir des conditions initiales; et si l'Univers contient une "surface fermée piégée ", c'est-à-dire une 2-surface dont tous les rayons lumineux émis convergent (voir, sur la figure 2, l'orientation des cônes de lumière), alors l'apparition d'une "singularité ", terminus de trajectoires de particules ou de rayons lumineux, est inévitable (voir l'encadré ci-dessus).

Résumé ainsi, en quoi ce théorème prouve-t-il que "la formation de trous noirs est une prédiction robuste de la 


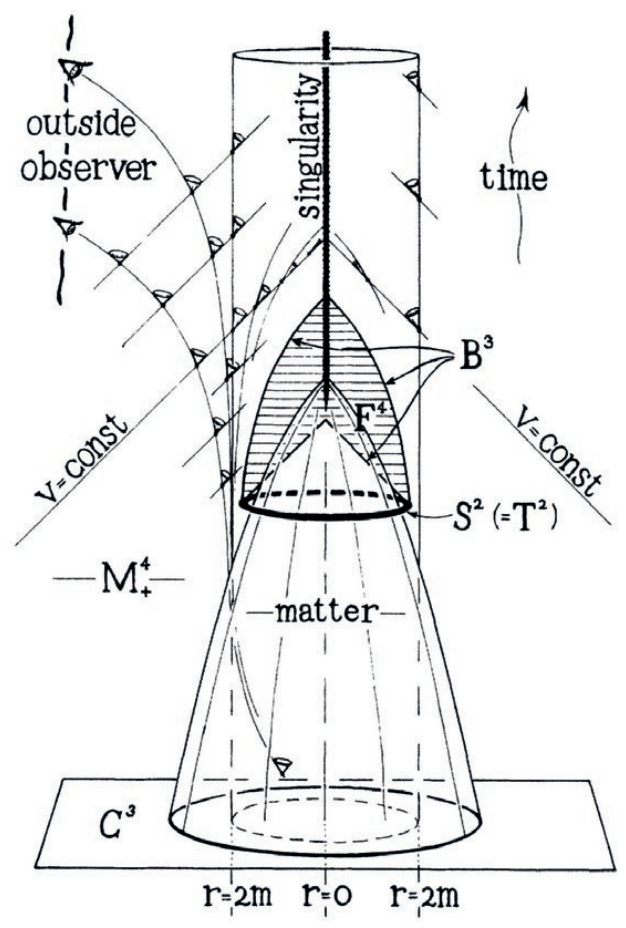

2. Le diagramme illustrant l'article de 1965 de Penrose [3]. II décrit l'effondrement d'une étoile à symétrie sphérique, c'est-à-dire la formation d'un trou noir " de Schwarzschild ». (Comme la démonstration du théorème n'utilise que des notions de topologie, voir encadré $\mathrm{p}$. 22 , il ne dépend pas de l'hypothèse de symétrie sphérique.)

Le 2-plan $C^{3}$ symbolise l'espace (courbé) qui décrit la gravitation à l'extérieur et à l'intérieur de l'étoile à l'instant initial $t=0$. Le cercle continu dessiné dans $C^{3}$ est la surface (bidimensionnelle) de l'étoile. L'axe vertical représente le temps. L'évolution de la matière sur le bord de l'étoile est représentée par la paire de lignes doubles qui montent et aboutissent en $r=0$ dans $\mathrm{F}^{4}$. Lorsque le temps croît, la taille de l'étoile diminue et franchit l'horizon $r=2 \mathrm{~m}$ du trou noir (dans le cas à symétrie sphérique). Le cercle en pointillés dans $\mathrm{C}^{3}$ est la " rétroprojection » (par homéomorphisme) dans $\mathrm{C}^{3}$ de la 3-surface de l'horizon (le « tube » vertical)

Le cercle $S^{2}\left(=T^{2}\right)$ est une " surface piégée ». Intuitivement : en l'absence de gravitation (i.e. dans l'espace-temps de Minkowski), si l'on émet de chaque point d'une 2-sphère des flashes de lumière vers l'intérieur, ils convergeront vers $r=0$ (en caustique) et, si on les émet vers l'extérieur, ils divergeront vers $r$ = l'infini. (Voir les cônes de lumière d'angle $90^{\circ}$ sur le diagramme, loin du centre où la gravité est négligeable.) Dans les régions où la gravité est plus forte, les cônes se resserrent et il peut arriver un moment où les flashes lumineux émis vers l'extérieur convergent eux aussi vers le centre (ainsi ils n'atteindront plus l'infini spatial) : ils sont piégés (sur le diagramme, c'est ce qu'il se passe quand $r$ devient inférieur à $2 \mathrm{~m}$ ). Le cercle $\mathrm{T}^{2}$ représente une telle 2 -surface piégée.

Dans le système de coordonnées choisi par Penrose, un côté des cônes de lumière (celui des rayons " entrants " à gauche du diagramme) reste tout le temps à $+45^{\circ}$; l'autre (celui des rayons " sortants ") est à $-45^{\circ}$ loin du centre, vertical sur l'horizon et à des angles positifs en deçà (l'angle d'ouverture des cônes s'amenuise).

Donc, la partie en pointillés (d'apex $90^{\circ}$ ) est l'avenir des rayons issus de $\mathrm{T}^{2}$ " entrants »; la partie supérieure en espèce de nez de fusée est l'avenir des rayons " sortants » (qui sont en fait piégés). Ainsi, la partie hachurée $\mathrm{F}^{4}$ (une portion d'espace-temps) est l'avenir de tous les rayons lumineux et de toute particule. $\mathrm{Et}^{3}$ (de dimension 3 ) est la frontière (tridimensionnelle) de $\mathrm{F}^{4}$.

\section{Pour en savoir plus}

1• S. Gillessen, [...], R. Genzel, "Monitoring Stellar Orbits Around the Massive Black Hole in the Galactic Center", The Astrophysical Journal, 692 (2009) 1075-1109.

2. A.M. Ghez et al., "Measuring Distance and Properties of the Milky Way's Central Supermassive Black Hole with Stellar Orbits", The Astrophysicial Journal, 689 (2008) 1044-1062.

3• R. Penrose, "Gravitational Collapse and Space-Time Singularities", Phys. Rev. Lett. 14 (1965) 57-59.
$>>$

théorie de la relativité générale " ? En quoi est-il si important qu'il mérite un prix Nobel ?

C'est parce qu'il est révolutionnaire.

Pour le démontrer, Penrose en effet a utilisé des notions et techniques de «topologie différentielle ", où l'on manipule des objets étendus (des surfaces par exemple) dont on étudie les propriétés globales (sont-elles fermées par exemple ?) et non les propriétés géométriques locales (qui distinguent une sphère d'un haricot). C'était la première fois que ces outils très puissants étaient utilisés en relativité générale (voir l'encadré p. 22). L'impact sur la discipline fut majeur.

Ainsi, Stephen Hawking développa immédiatement ces techniques pour, entre autres, débarrasser le théorème de certaines hypothèses trop fortes et préciser la nature des singularités : vu l'importance de ses contributions, il n'est pas absurde de concevoir qu'il aurait pu partager le prix s'il n'était mort en 2018. (Penrose et Hawking ont reçu le Prix Wolf en 1988 pour " leur brillant développement de la théorie de la relativité générale, grâce auquel ils ont montré l'inéluctabilité des singularités cosmologiques et ont élucidé la physique des trous noirs ".)

Ces notions nouvelles élargirent aussi profondément la «vision » que les physiciens se faisaient des trous noirs. On montra que l'aire de leurs bords ne pouvait qu'augmenter lors de leurs interactions, qu'il était donc envisageable de leur associer une entropie, une température, un rayonnement de corps noir.

On put aussi les concevoir comme des sortes de bulles de savon (de lumière, plus précisément), bulles qui interagissent, se déforment, fusionnent même. En exagérant à peine, les spectaculaires visualisations numériques, décrivant la fusion des deux trous noirs dont LIGO a observé le flash d'ondes gravitationnelles en 2015, reposent sur le théorème que Penrose avait démontré cinquante ans auparavant.

La relativité générale et les trous noirs ont été «nobélisés » en 2020, cent-cinq ans après leur invention. La physique est une aventure à long terme. D'autant qu'il reste à comprendre en quoi la théorie d'Einstein doit être modifiée pour éviter ces inéluctables singularités qu'elle prédit. 